\title{
BMJ Open What is the care pathway of patients who undergo thyroid surgery in France and its potential pitfalls? A national cohort
}

\author{
Muriel Mathonnet, ${ }^{1}$ Anne Cuerq, ${ }^{2}$ Christophe Tresallet, ${ }^{3}$ Jean-Christophe Thalabard, ${ }^{4}$ \\ Elisabeth Fery-Lemonnier, ${ }^{5}$ Gilles Russ, ${ }^{6}$ Laurence Leenhardt, ${ }^{6}$ Claude Bigorgne, ${ }^{7}$ \\ Philippe Tuppin, ${ }^{2}$ Bertrand Millat, ${ }^{8}$ Anne Fagot-Campagna ${ }^{2}$
}

To cite: Mathonnet M, Cuerq A, Tresallet C, et al. What is the care pathway of patients who undergo thyroid surgery in France and its potential pitfalls? A national cohort. BMJ Open 2017;7: e013589. doi:10.1136/ bmjopen-2016-013589

- Prepublication history for this paper is available online To view these files please visit the journal online (http://dx.doi.org/10.1136/ bmjopen-2016-013589).

Received 26 July 2016 Revised 6 January 2017 Accepted 11 January 2017

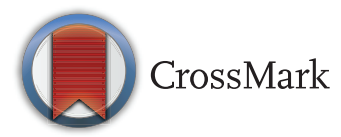

For numbered affiliations see end of article.

Correspondence to Dr Anne Fagot-Campagna; anne.fagot@cnamts.fr

\section{ABSTRACT}

Context: The rate of thyroid cancer is increasing in France, as well as concerns about overdiagnosis and treatment.

Objectives: To examine the care pathway of patients who undergo thyroid surgery in France and detect potential pitfalls.

Design: A large observational study based on medical reimbursements, 2009-2011.

Setting: Data from the Sniiram (National Health Insurance Information System).

Patients: Patients with thyroid surgery in 2010 , classified into 4 groups: thyroid cancer, benign nodule, goitre or multiple nodules, other (hyperthyroidism, head-neck cancer).

Main outcome measures: Medical investigations during, prior and after thyroidectomy.

Results: A total of 35367 patients underwent surgery (mean age 51 years, $80 \%$ women): $17 \%$ had a reported diagnosis of thyroid cancer, $20 \%$ benign nodule, $38 \%$ goitre or multiple nodules and $25 \%$ another diagnosis. The ratio of thyroidectomies with cancer over thyroidectomies with benign nodule was 0.8 and varied across regions. In the year preceding surgery, $82 \%$ of patients had an investigation by thyroid ultrasonography, $21 \%$ thyroid scintigraphy, $34 \%$ fine-needle aspiration cytology, $40 \%$ serum calcitonin assay and $54 \%$ serum calcium assay. In the following year, all patients with total thyroidectomy and $44 \%$ of patients with partial thyroidectomy and a diagnosis of benign nodule were taking thyroid hormone therapy. 100 patients had been reoperated for a compressive haematoma and 63 died during the first month, half of whom had been operated for cancer. Mean rates of recurrent laryngeal nerve injury and hypocalcaemia (requiring blood tests plus treatments within 4-12 months) were estimated at $1.5 \%$ and $3.4 \%$, respectively, and were higher in the cancer group $(2.3 \%$ and $5.7 \%)$.

Conclusions: This almost nationwide study demonstrates the suboptimal management of patients prior to thyroidectomy in France. It suggests overdiagnosis and potential harms to patients, and calls for a review of the relevance of thyroidectomy, particularly with regard to microcancers.
Strengths and limitations of this study

- The Sniiram database includes almost all the insured population in France where medical insurance is mandatory. It is one of the largest administrative databases. It has led to many publications to monitor quality of care, describe healthcare pathways at a national level and guide public health policies. In this paper, data from $77 \%$ of the French population were extracted to study the healthcare pathway of a cohort of 35000 people who underwent thyroidectomy in 2010 and provide a national picture.

- This observational study relies on the quality of the surgical procedure coding performed in public or private hospitals, as these procedure codes are necessary in order to classify the four groups: cancer, benign nodule, multiple nodules or goitre, and other cases. Misclassifications may have occurred, which are more likely to happen in the case of microcancers considered as benign nodules in our study.

- While the Sniiram database records the follow-up and reimbursements of all patients, we still may have missed some non-surgical investigations performed prior to surgery. We used a reasonable 1 -year interval to define this period but procedures performed during a public hospital stay are not systematically coded within the hospital when they do not provide higher funding to the hospital. However, procedures such as thyroid ultrasonography, scintigraphy, fine-needle aspiration cytology, serum calcitonin and calcium assays are rarely performed during a hospital stay.

- As the Sniiram does not provide outpatient diagnoses, we constructed several algorithms to define potential complications that did not require systematic hospitalisation, such as hypoparathyroidism (defined with more than three serum calcium assays and three deliveries of calcium supplements during the 4th to the 12th month after thyroidectomy) or recurrent laryngeal nerve injury (several definition based on speech therapy sessions, ear-nose-throat specialist visits or functional testing during the first 12 postoperative months). 


\section{INTRODUCTION}

The prevalence of all forms of thyroid disease is difficult to assess. Published clinical trials are often old and the performances of detection have substantially improved over time, ${ }^{1}<\mathrm{QC}$ : There are 26 refs in the footnotes, which I have edited. Please check with the PE if the corrections are correct.>consequently modifying patient management. In countries with sufficient dietary iodine intake, such as France or the USA, the clinical prevalence of thyroid nodules is about $5 \%$ and is higher in women (5.3-6.4\% vs $0.8-1.6 \%$ for men) and persons over the age of 50 years in whom the prevalence is about $30-40 \% .^{2}$ The prevalence of thyroid disease based on ultrasonography screening is much higher and is currently estimated to be $67 \%,{ }^{13}$ comparable to the rate of nodules discovered at autopsy. ${ }^{13} 4$

The growing prevalence of thyroid cancer has been clearly established. Over the past three decades, the number of new cases diagnosed in France has increased fivefold in both sexes. ${ }^{5} 6$ This increased prevalence almost exclusively concerns papillary cancers, with no impact on mortality, which has decreased over the same period. Over a period of 20 years, the proportion of microcancers $(<10 \mathrm{~mm})$ has increased from $4 \%$ to more than $50 \%$. One-half of these microcancers are smaller than $3 \mathrm{~mm}$ and are discovered incidentally on thyroidectomy specimens. This increased prevalence of microcancers is directly related to progress in the detection of nodules by increasingly efficient ultrasound machines and progress in the histological diagnosis of cancer as a result of very thin histological sections and the use of immunohistochemistry. In fact, the majority of these microcancers appear to undergo growth arrest, while progression to symptomatic cancer is observed in only 1 out of every 15 nodules. The increased incidence of thyroid cancer is thus due to microcancers and can be considered to constitute a form of overdiagnosis. ${ }^{7}$ The diagnosis of thyroid cancer, regardless of stage, results in an alteration of the patient's quality of life and social representation and can be responsible for sometimes unjustified modifications of therapeutic management or potentially morbid treatment follow-up, resulting in increased costs induced by incidentally discovered cancers.

Over the past 10 years, several medical authorities have published guidelines for the management of thyroid nodules and/or thyroid cancers: the European Thyroid Association (ETA) and the American Association of Clinical Endocrinologists (AACE) in 2006, the British Thyroid Association (BTA) in 2007, the National Cancer Institute (NCI) in 2008, the American Thyroid Association (ATA) in 2009 and 2015, and the Société Française d'Endocrinologie (SFE) in 2011. Despite several differences, all medical societies recommend thyroid-stimuling hormone (TSH) assay and thyroid ultrasonography in all patients with thyroid disease combined with fine-needle aspiration cytology of nodules with features suggestive of malignancy. ${ }^{8-10}$ According to the SFE, the majority of thyroid incidentalomas require simple surveillance. The indications for thyroid surgery are rare, limited to nodules demonstrated to be malignant on preoperative investigations and very large or retrosternal nodules, ${ }^{10}$ symptomatic or unsightly goitre or goitre accompanied by low TSH. Despite existing international and national recommendations, much concern is being currently raised about overdiagnosis and an excess of thyroidectomy, which may result in harms to the patients.

The objective of this observational study was to analyse the care pathway of patients prior to thyroidectomy in France during the year 2010 and to study the impact of surgery on postoperative morbidity and mortality in the Sniiram (French National Health Insurance Information System) database, a largely published and nationwide comprehensive administrative database of about 56 million people based on medical reimbursement data. ${ }^{11}$

\section{METHODS}

\section{Information system and population}

In France, the Sniiram is an anonymous, individual database concerning all the beneficiaries of the various national health insurance schemes. ${ }^{1-13}$ Medical insurance is mandatory and is provided by the State for lowincome people. Many published studies have been based on the Sniiram which stands among the largest medicoadministrative databases worldwide and is largely used to guide public health policies in France as these data allow the systematic follow-up of all medical care received by the population. ${ }^{12} 13$ It exhaustively records all reimbursed prescriptions and outpatient services and procedures, as well as their date, over the previous 3 years plus the current year. Identification of medicinal products is based on the Anatomical Therapeutic Classification (ATC) code, that of laboratory examinations is based on the national laboratory test coding table and that of procedures is based on the Classification Commune des Actes Médicaux (CCAM; common classification of medical procedures). The Sniiram does not contain any clinical data concerning the results related to prescriptions or examinations, but it nevertheless includes information on the possible presence of long-term diseases (LTDs), such as cancers, eligible for $100 \%$ reimbursement of healthcare expenditure following approval by a national health insurance physician. These LTDs are coded according to the International Classification of Diseases (ICD-10). A unique and anonymous identification number for each person also allows integration into the Sniiram database of the hospital discharge database (PMSI, Programme de médicalisation des systèmes d'information). The principal diagnoses and associated diagnoses recorded in the PMSI are coded according to the ICD-10 and the procedures performed, such as thyroidectomies, are coded according to the CCAM.

In 2010, the national health insurance general scheme (excluding local mutualist sections that provide medical 
insurance for, eg, students, teachers) covered about $77 \%$ of the 65 million inhabitants in France including lowincome people and was the only scheme for which both vital status and LTDs were comprehensively recorded at that time. Data for the health insurance general scheme beneficiaries who underwent thyroid surgery in 2010 were extracted from the Sniiram database. The diagnoses recorded during the hospital stay, and the clinical examinations and complementary investigations performed 1 year before and 1 year after surgery, estimated by reimbursement data, were analysed. In order to establish an estimate for France as a whole, the sample sizes of general scheme beneficiaries undergoing thyroid surgery were extrapolated (by age group and gender) to the national estimates provided by Insee (Institut national de la statistique et des études économiques) for the total population of France in January 2011. For the purposes of regional comparisons, regional rates of the general scheme were standardised for the age and gender structure of the Insee total population of France in January 2011.

Patients who had undergone thyroidectomy were classified into four exclusive groups according to the type of thyroid disease. The first group was composed of patients who had a diagnosis of thyroid cancer recorded in the databases. This group included patients with ICD-10 codes for malignant neoplasm of thyroid gland (C73, D09.3), neoplasm of uncertain behaviour of thyroid gland (D44.0), hypersecretion of calcitonin (E07.0) or multiple endocrine adenomatosis (D44.8) coded during the hospital stay for thyroidectomy or in the LTD coding, and patients who underwent lymph node dissection or radioiodine therapy without a diagnosis of hyperthyroidism.

The second group was composed of patients who had a recorded diagnosis of benign nodule according to ICD-10 codes for non-toxic single thyroid nodule (E04.1), benign nodule of the thyroid gland (D34) or benign tumour of other and unspecified endocrine glands (D35.7, D35.8, D35.9) during the hospital stay.

The third group was composed of patients who had a recorded diagnosis of goitre or multiple nodules on the basis of ICD-10 codes for non-toxic diffuse goitre (E04.0), non-toxic multinodular goitre (E04.2), other specified non-toxic goitre (E04.8), non-toxic goitre, unspecified (E04.9), congenital hypothyroidism with diffuse goitre (E03.0), dyshormogenetic goitre (E07.1), or iodine deficiency-related goitre (E01.0, E01.1, E01.2).

Finally, the fourth group comprised patients who had another recorded diagnosis, especially head and neck cancer and hyperthyroidism. These patients were excluded from the subsequent analysis, as thyroidectomy was simply an associated procedure or was performed for hyperthyroidism.

\section{Definitions and statistical analysis}

The care pathway was analysed in rolling years, 12 months before and 12 months after the date of thyroidectomy. A thyroidectomy frequency ratio was calculated between group 1 (cancer) and group 2 (benign nodule), overall and by region. In order to study regional variability, data were standardised for the age and gender of the population of beneficiaries on 31 December 2010. We compared the lowest to the highest value of 25 French regions using $\chi^{2}$ tests. The 26th region (Guyana) was excluded due to a small number of cases ( 7 cases of cancer and 19 benign nodules) and different care pathways occurring in this rural region. Drug treatments were identified by the presence of at least three reimbursements over the 12-month period before and then the 12-month period after hospitalisation. Thyroid ultrasonography, fine-needle aspiration cytology and scintigraphy were identified by the presence of specific codes, whether they were performed in a hospital outpatient department or in private practice. However, the procedures performed during a public hospital stay were not systematically coded at that time, possibly resulting in missing data. Similarly, laboratory tests performed during a public hospital stay were not identified, as they are not reimbursed individually. Reimbursements for hospital outpatient and private practice endocrinology and visits to an ear-nose-throat (ENT) specialist were taken into account. There again, ambulatory visits to a public hospital specialist were not systematically coded at that time, possibly resulting in missing data. Postoperative sick leave allowances were also taken into account.

Complications were identified during the thyroidectomy hospital stay and over the following year from hospitalisation and/or ambulatory reimbursement data. Severe complications were defined by the development of compressive haematoma during the thyroidectomy hospital stay, death (in-hospital or during the first month) or the presence of a CCAM procedure supposedly related to thyroidectomy (tracheobronchial stent, tracheotomy, arytenoidectomy, etc). Readmissions for thyroid problems or for phosphorus-calcium imbalance were also identified. Various indicators that could also constitute markers of late ENT complications were constructed: at least two visits to an ENT specialist, at least one visit to a speech therapist, laryngeal function tests looking for recurrent laryngeal nerve injury, laryngoscopy, etc, over the 12-month period. Hypoparathyroidism was suspected by the presence of at least three serum calcium assays and at least three deliveries of calcium supplements over the period ranging from 4 to 12 months after surgery (to avoid selecting very transient hypoparathyroidism), or the presence of hospitalisation with a diagnosis of hypoparathyroidism over the 12-month period.

Finally, the number of patients who had undergone thyroidectomy and those with LTDs for thyroid cancer from 2010 to 2013 were analysed in order to estimate temporal trends.

Statistical analyses were performed with SAS software (SAS Enterprise Guide, V.4.3, SAS Institute, Cary, North Carolina, USA). Analyses of the Sniiram database have 
been approved by the French personal data protection agency (Commission Nationale Informatique et Libertés). Since the Sniiram database is anonymous, no other ethical approval was required for this study.

\section{RESULTS}

Among 50 million people insured under the health insurance general scheme ( $77 \%$ of the French population), 35367 underwent thyroid surgery in 2010, that is, by extrapolation based on the age and gender structure of the French population, about 45800 people in the overall French population. Patient characteristics and characteristics of the surgical procedures performed are reported in table 1 .

Patients with a diagnosis of multiple nodules or goitre represented the largest subgroup (38\% of patients), followed by those with a benign nodule $(20 \%)$ and those with thyroid cancer, regardless of the stage (17\%). One-quarter of patients had another type of diagnosis, mainly head and neck cancer or hyperthyroidism. Each of these subgroups comprised $80 \%$ women with a mean age of 51 years. Total thyroidectomy (or completion thyroidectomy) was performed for about $89 \%$ of patients with a postoperative diagnosis of thyroid cancer and $86 \%$ of cases of multiple nodules or goitres, while partial thyroidectomy was performed in $71 \%$ of patients with benign nodules. Patients were more frequently operated in private or public hospitals with high thyroid surgery rates, especially when they had a diagnosis of thyroid cancer or multiple nodules or goitres.

The rate of thyroidectomy with a diagnosis reported as thyroid cancer, nodule or goitre in 2010 (excluding thyroidectomies for head and neck cancer or hyperthyroidism) was 5.3 per 10000 inhabitants and the standardised rates varied according to regions between 4.0 and 8.1 per 10000 inhabitants $(p=0.003)$ as shown in figure 1 .

In patients 20 years and older, the ratio of the number of thyroidectomies with a diagnosis of cancer over the number of thyroidectomies with a diagnosis of benign nodule was 0.8 . This ratio varied between regions from 0.5 in Basse-Normandie, Bretagne, Limousin and Languedoc-Roussillon to 2.6 in Nord-Pas-de-Calais, as shown in figure 2. The percentage of thyroidectomies with a diagnosis of cancer over the total number of thyroidectomies with a diagnosis of cancer or benign nodule varied significantly from $28 \%$ to $69 \%$ ( $\mathrm{p}=0.001$ ).

During the year preceding thyroid surgery, healthcare varied according to the group. Eighty per cent of patients of group $1(n=5979)$ who finally had a diagnosis of thyroid cancer had evidence of investigation by thyroid ultrasonography and $44 \%$ by fine-needle aspiration cytology (table 2) prior to surgery. In group 2 $(n=7270)$, corresponding to patients who had a recorded diagnosis of benign nodule, the fine-needle aspiration cytology rate was $34 \%$.

Among people with thyroidectomy and a diagnosis of cancer or benign nodule, the overall fine-needle aspiration cytology rate was $39 \%$ and the standardised rates varied according to regions between $11 \%$ (Franche-Comté) and $53 \%$ (Ile de France; $p=0.001$, figure 3 ).

In the three groups of patients, TSH assays had been performed in about $90 \%$ of patients, $\mathrm{T} 4$ assay in more than $63 \%$, T3 assay in more than $35 \%$ and a thyroid scintigraphy in more than $18 \%$, prior to surgery. Serum calcitonin assay had been performed in $44 \%$ of patients and serum calcium assay in $58 \%$ of patients who finally had a diagnosis of thyroid cancer. These proportions were $39 \%$ and $50 \%$, respectively, for patients with a diagnosis of benign nodule. Less than one-half of patients, regardless of their thyroid disease, were referred to an endocrinologist. Finally, neither thyroid ultrasonography nor fine-needle aspiration cytology was performed in $10 \%$ of patients, neither T3, T4 nor TSH assay was performed in about $9 \%$ of patients in groups 1 and 2 .

The fine-needle aspiration cytology rate varied according to the region and was probably related to the availability of doctors able to perform this technique and cytopathologists. Among the patients who had undergone surgery and had a diagnosis of thyroid cancer or

Table 1 Characteristics of the 35367 health insurance general scheme beneficiaries who underwent thyroidectomy in 2010, by the type of thyroid diagnosis, Sniiram, France

\begin{tabular}{|c|c|c|c|c|}
\hline & $\begin{array}{l}\text { Cancer } \\
\mathrm{N}=5979 \\
(17 \%)\end{array}$ & $\begin{array}{l}\text { Benign nodule } \\
\mathrm{N}=7270 \\
(20 \%)\end{array}$ & $\begin{array}{l}\text { Multiple nodules and goitre } \\
\mathrm{N}=13350 \\
(38 \%)\end{array}$ & $\begin{array}{l}\text { Others } \\
\mathrm{N}=8768 \\
(25 \%)\end{array}$ \\
\hline Age $<20$ years & $1.4 \%$ & $1.6 \%$ & $0.4 \%$ & $1.0 \%$ \\
\hline Age $<50$ years & $47 \%$ & $53 \%$ & $40 \%$ & $44 \%$ \\
\hline Women & $76 \%$ & $79 \%$ & $83 \%$ & $79 \%$ \\
\hline \multicolumn{5}{|l|}{ Thyroidectomy } \\
\hline Total or subtotal & $77 \%$ & $26 \%$ & $85 \%$ & $79 \%$ \\
\hline Partial & $11 \%$ & $71 \%$ & $12 \%$ & $18 \%$ \\
\hline Completion & $12 \%$ & $3 \%$ & $3 \%$ & $3 \%$ \\
\hline Surgery performed in a public hospital & $54 \%$ & $38 \%$ & $46 \%$ & $54 \%$ \\
\hline \multicolumn{5}{|c|}{ Surgery practiced in a public or private hospital that performed } \\
\hline$<30$ thyroid surgeries/year & $16 \%$ & $24 \%$ & $17 \%$ & $15 \%$ \\
\hline$>100$ thyroid surgeries/year & $51 \%$ & $37 \%$ & $41 \%$ & $55 \%$ \\
\hline
\end{tabular}


Figure 1 Age and gender standardised rates of patients with thyroidectomy, by region, in 2010, Sniiram, France.

Figure 2 Ratios of the number of thyroidectomies with a diagnosis of cancer over the number of thyroidectomies with a diagnosis of benign nodule, by region, in 2010, Sniiram, France.

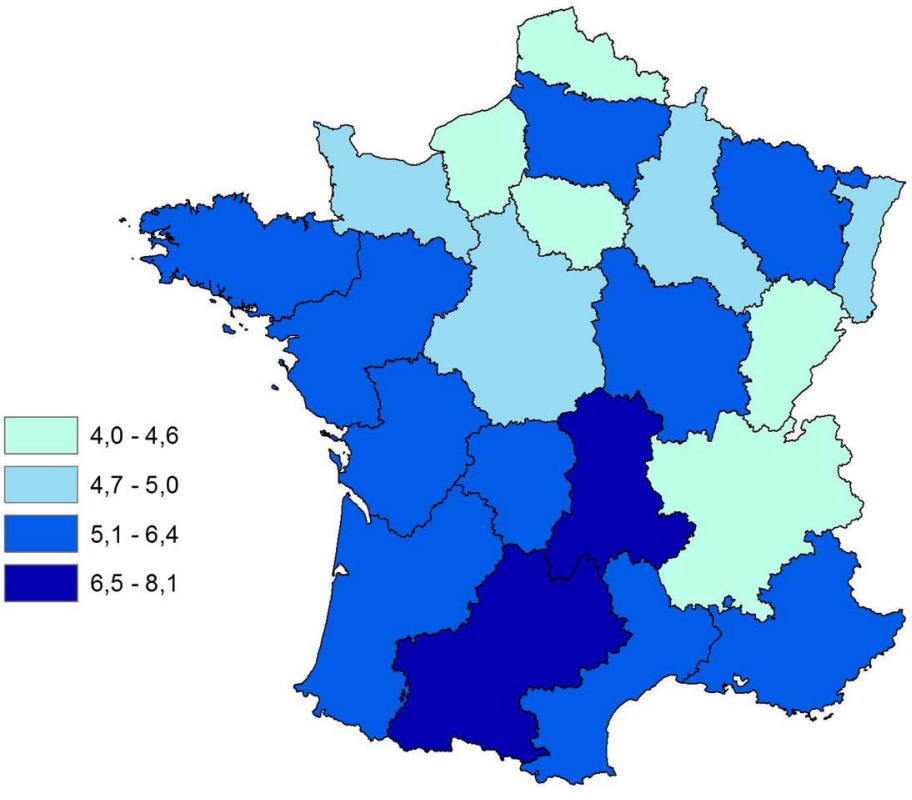

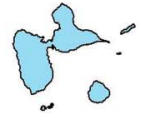

Guadeloupe

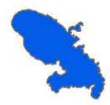

Martinique

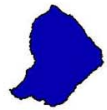

Guyane

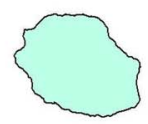

Réunion

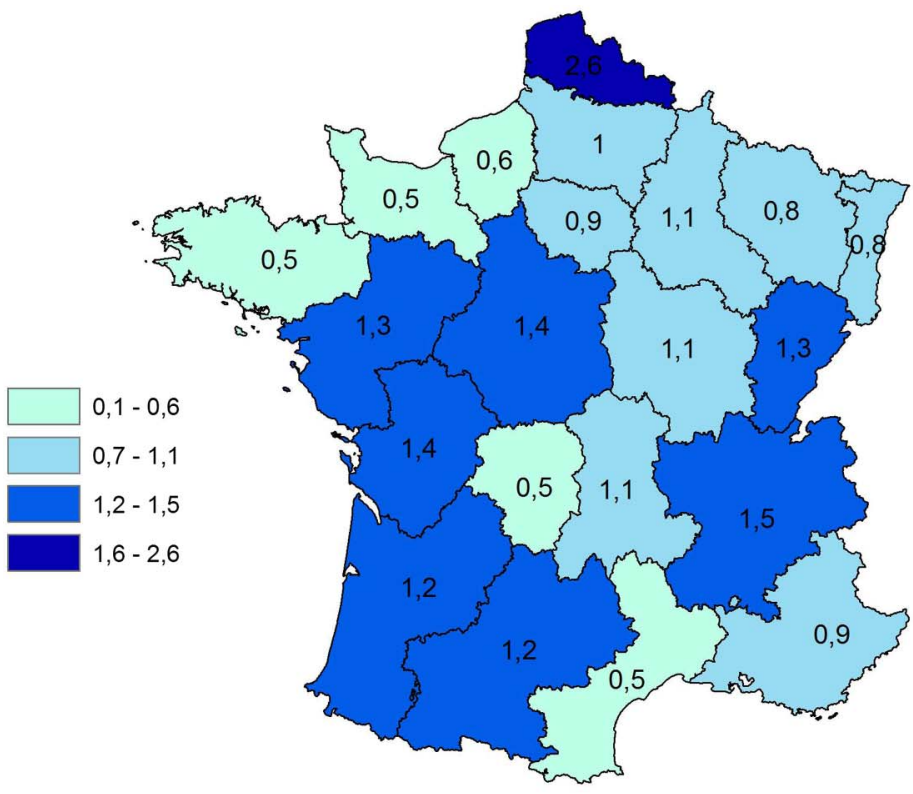

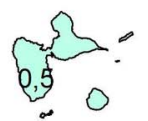

Guadeloupe

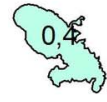

Martinique

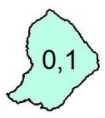

Guyane
0,4

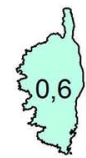


Table 2 Prethyroidectomy care pathway (previous 12 months prior surgery in 2010), by the type of thyroid diagnosis, Sniiram, France

\begin{tabular}{|c|c|c|c|c|c|}
\hline & $\begin{array}{l}\text { Cancer } \\
\mathrm{N}=5979\end{array}$ & $\begin{array}{l}\text { Benign } \\
\text { nodule } \\
\mathrm{N}=7270\end{array}$ & $\begin{array}{l}\text { Multiple nodules } \\
\text { and goitre } \\
\mathrm{N}=13350\end{array}$ & $\begin{array}{l}\text { Cancer/ } \\
\text { benign } \\
\text { nodule ratio }\end{array}$ & $\begin{array}{l}\text { Cancer/ } \\
\text { goitre ratio }\end{array}$ \\
\hline Thyroid ultrasound & $80 \%$ & $84 \%$ & $82 \%$ & 1.0 & 1.0 \\
\hline Fine-needle aspiration cytology of the thyroid & $44 \%$ & $34 \%$ & $23 \%$ & 1.3 & 1.9 \\
\hline Thyroid scintigraphy & $18 \%$ & $21 \%$ & $22 \%$ & 0.9 & 0.8 \\
\hline At least one of the 3 & $89 \%$ & $91 \%$ & $88 \%$ & 1.0 & 1.0 \\
\hline TSH assay & $89 \%$ & $91 \%$ & $92 \%$ & 1.0 & 1.0 \\
\hline T3 assay & $36 \%$ & $35 \%$ & $37 \%$ & 1.0 & 1.0 \\
\hline T4 assay & $63 \%$ & $65 \%$ & $66 \%$ & 1.0 & 1.0 \\
\hline Calcitonin assay & $44 \%$ & $39 \%$ & $39 \%$ & 1.1 & 1.1 \\
\hline Calcium assay & $58 \%$ & $50 \%$ & $55 \%$ & 1.2 & 1.1 \\
\hline No T3, T4 or TSH assay & $10 \%$ & $8 \%$ & $8 \%$ & 1.2 & 1.3 \\
\hline$\geq 3$ thyroid hormone deliveries & $21 \%$ & $16 \%$ & $19 \%$ & 1.3 & 1.1 \\
\hline$\geq 1$ endocrinology consultation & $49 \%$ & $44 \%$ & $49 \%$ & 1.1 & 1.0 \\
\hline$\geq 1$ ear-nose-throat specialist consultation & $43 \%$ & $49 \%$ & $43 \%$ & 0.9 & 1.0 \\
\hline
\end{tabular}

Figure 3 Fine-needle aspiration cytology standardised rates among people with thyroidectomy and a diagnosis of cancer or benign nodule, by region, in 2010, Sniiram, France.

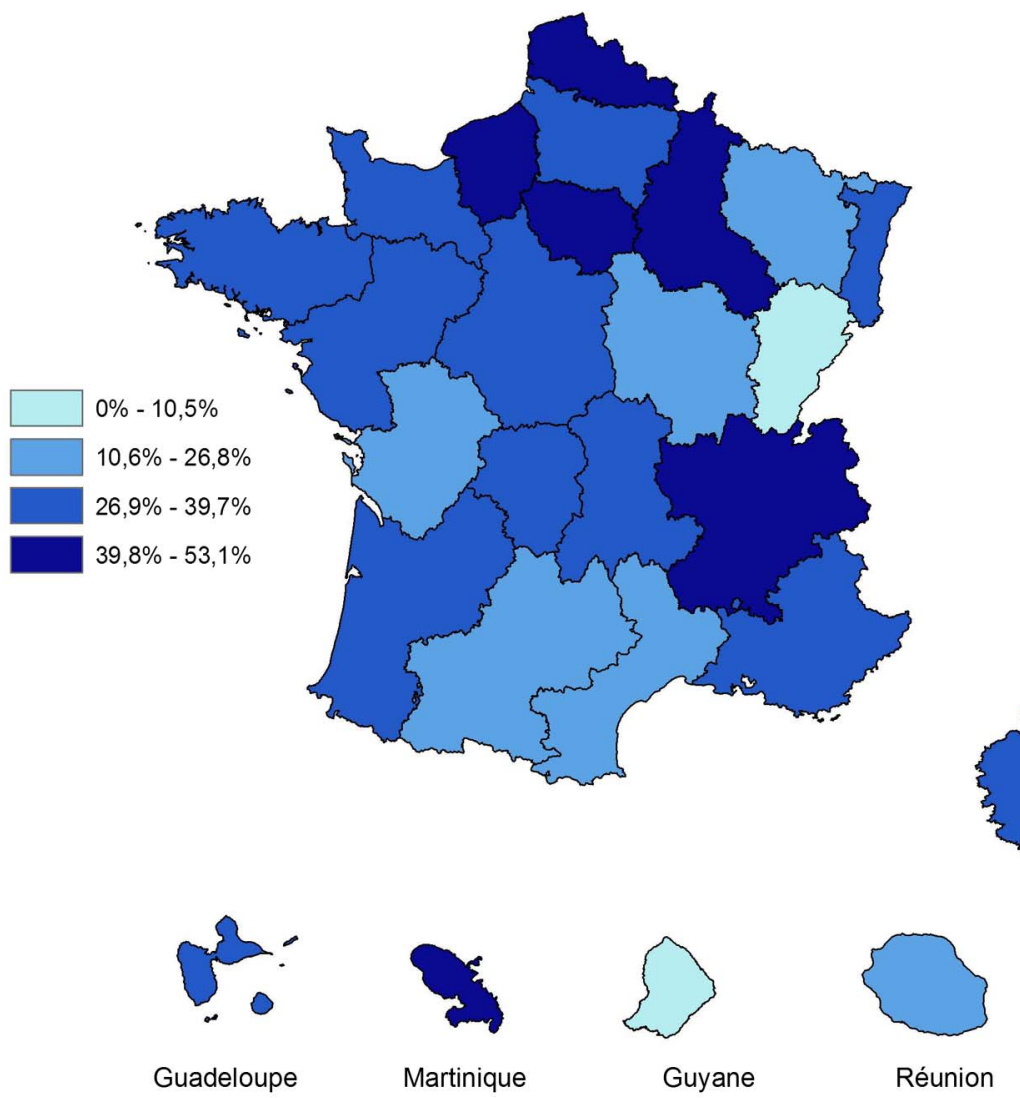

benign nodule, the fine-needle aspiration cytology rate was $53 \%$ in patients from Ile-de-France and Rhône-Alpes, but only $10 \%$ in those from Franche-Comté (and $0 \%$ in Guyana for only 28 patients undergoing surgery). The regional rates of fine-needle aspiration cytology were significantly correlated with the regional rates of thyroidectomy (Spearman correlation coefficient test: $\mathrm{r}=0.48$, $\mathrm{p}=0.034)$.

During the 12 months following surgery, TSH assay was performed in almost all patients (table 3). T3 assay was performed in $51 \%$ of patients with thyroid cancer, while the rate of total thyroidectomy in this group was $76 \%$. T3 assay was also performed in $26 \%$ of patients with a diagnosis of benign nodule, but the rate of partial thyroidectomy in this group was $71 \%$. Thyroid hormone replacement therapy was administered to all patients who had undergone total thyroidectomy and in $44 \%$ of patients who had undergone partial thyroidectomy and had a diagnosis of benign nodule. The endocrinologist referral rate remained low: $56 \%$ of patients with thyroid 
cancer and $34 \%$ of patients with benign nodule consulted an endocrinologist. The mean duration of sick leave for employed patients was 89 days for patients with thyroid cancer and 38 days for patients with benign nodules. Sick leave lasted more than 3 weeks in $60-81 \%$ of cases, depending on the group.
Severe complications of thyroid surgery were rare (table 4). About 20 patients, 14 in the cancer group and $<10$ in the multiple nodules and goitre group (none in the benign nodule group), died in hospital; another 11 patients died during the first 30 days after surgery, that is, an overall short-term mortality of about

Table 3 Post-thyroidectomy care pathway (following 12 months after surgery in 2010), by the type of thyroid diagnosis, Sniiram, France

\begin{tabular}{|c|c|c|c|c|c|}
\hline & $\begin{array}{l}\text { Cancer } \\
\mathrm{N}=5979\end{array}$ & $\begin{array}{l}\text { Benign } \\
\text { nodule } \\
\mathrm{N}=7270\end{array}$ & $\begin{array}{l}\text { Multiple nodules } \\
\text { and goitre } \\
\mathrm{N}=13350\end{array}$ & $\begin{array}{l}\text { Cancer/benign } \\
\text { nodule ratio }\end{array}$ & $\begin{array}{l}\text { Cancer/goitre } \\
\text { ratio }\end{array}$ \\
\hline TSH assay & $96 \%$ & $93 \%$ & $97 \%$ & 1.0 & 1.0 \\
\hline T3 assay & $51 \%$ & $26 \%$ & $28 \%$ & 2.0 & 1.9 \\
\hline T4 assay & $76 \%$ & $63 \%$ & $68 \%$ & 1.2 & 1.1 \\
\hline Levothyroxine replacement therapy & $95 \%$ & $58 \%$ & $92 \%$ & 1.6 & 1.0 \\
\hline In those with total thyroidectomy & $99 \%$ & $97 \%$ & $99 \%$ & 1.0 & 1.0 \\
\hline In those with partial thyroidectomy & $73 \%$ & $44 \%$ & $48 \%$ & 1.7 & 1.5 \\
\hline$\geq 1$ endocrinology consultation & $56 \%$ & $34 \%$ & $39 \%$ & 1.7 & 1.4 \\
\hline$\geq 1$ ear-nose-throat specialist consultation & $33 \%$ & $36 \%$ & $32 \%$ & 0.9 & 1.0 \\
\hline \multicolumn{6}{|l|}{ In patients with at least one day of sick leave } \\
\hline Mean number of days/patient & 89 days & 38 days & 45 days & 2.3 & 2.0 \\
\hline \multicolumn{6}{|l|}{ Per cent of patients, with } \\
\hline$>10$ days of sick leave & $96 \%$ & $94 \%$ & $97 \%$ & 1.0 & 1.0 \\
\hline$>14$ days of sick leave & $93 \%$ & $85 \%$ & $93 \%$ & 1.1 & 1.0 \\
\hline$>21$ days of sick leave & $81 \%$ & $60 \%$ & $75 \%$ & 1.4 & 1.1 \\
\hline
\end{tabular}

Table 4 Immediate and late complications of thyroidectomy performed in 2010, by the type of thyroid diagnosis, Sniiram, France

\begin{tabular}{|c|c|c|c|c|c|}
\hline & $\begin{array}{l}\text { Cancer } \\
\mathrm{N}=5979\end{array}$ & $\begin{array}{l}\text { Benign } \\
\text { nodule } \\
\mathrm{N}=7270\end{array}$ & $\begin{array}{l}\text { Multiple } \\
\text { nodules } \\
\text { and goitre } \\
\mathrm{N}=13350\end{array}$ & $\begin{array}{l}\text { Cancer/ } \\
\text { benign } \\
\text { nodule ratio }\end{array}$ & $\begin{array}{l}\text { Cancer/ } \\
\text { goitre } \\
\text { ratio }\end{array}$ \\
\hline \multicolumn{6}{|l|}{ Immediate complications } \\
\hline \multicolumn{6}{|l|}{ During the hospitalisation } \\
\hline Mean length of stay $>3$ days & $42 \%$ & $29 \%$ & $39 \%$ & 1.4 & 1.1 \\
\hline One-month mortality & $0.3 \%(n=19)$ & $0.0 \%(n<10)$ & $0.1 \%(n=10)$ & - & - \\
\hline \multicolumn{6}{|l|}{ Late complications } \\
\hline Severe complications* & $1.4 \%(n=84)$ & $0.4 \%(n=32)$ & $0.6 \%(n=86)$ & 3.5 & 2.3 \\
\hline \multicolumn{6}{|l|}{ Other complications during the first year } \\
\hline At least 2 ear-nose-throat specialist consultations & $15 \%$ & $13 \%$ & $11 \%$ & 1.2 & 1.3 \\
\hline $\begin{array}{l}\text { At least } 3 \text { serum calcium assays and at least } 3 \\
\text { deliveries of calcium supplements } \ddagger\end{array}$ & $5.7 \%$ & $1.0 \%$ & $3.5 \%$ & 5.7 & 1.6 \\
\hline Readmission for hypoparathyroidism & $0.2 \%(n=11)$ & $0 \%(n=0)$ & $0 \%(n=0)$ & - & - \\
\hline At least 1 hypoparathyroidism complication & $5.8 \%$ & $1.0 \%$ & $3.5 \%$ & 5.8 & 1.7 \\
\hline At least 1 complication or hypoparathyroidism & $27 \%$ & $18 \%$ & $20 \%$ & 1.5 & 1.3 \\
\hline Other readmissions for-thyroid disorders & $6.4 \%$ & $1.4 \%$ & $2.2 \%$ & 4.6 & 2.9 \\
\hline Hypercalcaemia & $1.2 \%(n=71)$ & $0.1 \%(n<10)$ & $0.1 \%(n=18)$ & - & - \\
\hline
\end{tabular}


30 patients. The cause of death is not indicated in these administrative databases. One hundred patients, 25 in the cancer group and 75 all together in the benign nodule group and the goitre group, experienced postoperative compressive haematoma requiring reoperation. This compressive haematoma rate $(0.4 \%)$ did not appear to be related to the underlying thyroid disease or to the type of surgical procedure performed, such as radical thyroidectomy or lymph node dissection.

The late complication rate was estimated by the number of specialist visits or procedures, or the readmission rate during the year following surgery. Patients with more than two ENT or speech therapy visits and patients in whom a laryngeal procedure was performed were considered to have experienced a laryngeal complication. The late complication rate varied according to the group from $17 \%$ to $23 \%$, and the recurrent laryngeal nerve injury rate varied from $2.3 \%$ to $1.2 \%$. Patients in whom more than three serum calcium assays were performed and to whom calcium supplements were dispensed more than three times during the 4th to the 12th month following surgery were considered to suffer from persistent hypoparathyroidism. This hypoparathyroidism rate ranged from $5.7 \%$ for the thyroid cancer group to $1 \%$ for the nodule group. Among people with a diagnosis of benign nodule, a marker of hypoparathyroidism was recorded in 10 persons $(0.2 \%)$ who underwent partial thyroidectomy and $63(3 \%)$ of those with total or subtotal thyroidectomy. The readmission rate in the thyroid cancer group was higher for hypercalcaemia than for hypocalcaemia: $1.2 \%$ and $0.2 \%$, respectively.

Between 2010 and 2012, the number of patients who had undergone thyroidectomy increased by 400 patients each year, from about 35400 in 2010 to 36200 in 2012, that is, a mean annual growth rate of $+1.1 \%$. This trend was reversed between 2012 and 2013, as the number of patients decreased by 900 patients to 35300 , that is, a growth rate of $-2.6 \%$. The number of patients with LTD $100 \%$ health insurance cover for thyroid cancer increased from 63311 in 2011, to 65401 in 2012 and 67461 in 2013, that is, a mean annual growth rate of $+3.2 \%$. In parallel, between 2010 and 2013 among all general scheme beneficiaries, the number of thyroid ultrasonography examinations performed increased from 1.12 to 1.19 million $(+2.2 \%$ /year $)$ and the number of thyroid fine-needle aspiration cytology procedures increased from 89000 to $98000(+3.4 \% /$ year $)$, while the number of thyroid scintigraphies decreased from 66000 to $58500(-3.8 \% /$ year $)$. The number of TSH assays (alone or combined with other parameters) increased from 12.2 to 14.8 million (+7\%/year), while the number of free T4 assays (alone or combined with other parameters) increased from 3.0 to 3.6 million $(+7 \% /$ year), and the number of free T3 assays (alone or combined with other parameters) also increased from 1.0 to 1.3 million $(+10 \% /$ year $)$.

\section{DISCUSSION}

This observational and almost nationwide study based on more than 35000 patients first demonstrates the suboptimal management of patients prior to thyroidectomy in France. The thyroidectomy rate with a diagnosis of benign nodule appears to be excessively high compared with the thyroidectomy rate with a diagnosis of thyroid cancer. Furthermore, these rates vary considerably from one region to another, documenting variations in clinical practices across the country. Fine-needle aspiration cytology before surgery for a suspicious thyroid nodule is performed in less than one-half of cases, while this procedure could avoid surgery for a certain number of patients, which, as shown by this study as well as other studies, is not devoid of complications. Second, some examinations are performed too frequently, such as preoperative thyroid scintigraphy and T4 assay, and preoperative and postoperative T3 assay. Therefore, these data suggest that suboptimal management prior to thyroidectomy leads to overdiagnosis and potential harms to patients ${ }^{7}$ as well as a lack of efficiency for the medical insurance system.

The volume of data collected from a database covering $77 \%$ of the French population allows us to analyse the healthcare pathway and evaluate its health impact. ${ }^{11-13}$ Although guidelines ${ }^{8-10}$ do not specify a maximum interval between preoperative assessment and thyroid surgery, the 1-year interval adopted for this study appears to be reasonable. Some non-surgical procedures considered to be necessary to the preoperative and postoperative care pathways may not have been identified from the Sniiram database when they were performed during a public hospital stay as they are not systematically coded within the hospital when they do not provide higher funding to the hospital. However, these nonsurgical procedures to investigate thyroid disease are rarely performed during a hospital stay. More importantly, the quality of this study relies on the quality of the surgical procedure coding performed in public or private hospitals, as these procedure codes are necessary in order to classify the four groups of thyroidectomies: cancer, benign nodule, multiple nodules or goitre, other cases. Other data were therefore investigated, such as requests for LTD coverage for thyroid cancer, reimbursement for radioiodine therapy or lymph node dissection, to isolate cancer cases. Nevertheless, it is still possible that the group described as surgery with a diagnosis of benign nodule included few cases of thyroid cancer, which are more likely to be microcancers. Our data set also does not provide information on cancer size. However, the incidence of thyroid cancers and especially the incidence of micropapillary carcinomas has been studied based on a French registry covering over 3000 cases of thyroid carcinomas and a population over 4.6 million inhabitants. ${ }^{6}$ During the period 1998-2000, half of thyroid carcinomas diagnosed had a size smaller than $10 \mathrm{~mm}$ and a third $5 \mathrm{~mm}$ or less. Between the years 1983-1985 and 1998-2000, the number of tumours with 
a size smaller than $10 \mathrm{~mm}$ increased ninefold over the same period.

Despite a number of differences, all medical societies recommend TSH assay and thyroid ultrasonography, as a basic work-up in patients with any form of thyroid disease, and a fine needle-aspiration cytology, guided by the ultrasound feature of nodule guidelines. ${ }^{8-10}$ Our analyses show that the thyroidectomy rate started to decline in France between 2012 and 2013 (-900 thyroidectomies), although the number of patients with $100 \%$ fee coverage for thyroid cancer as LTD continued to increase. Therefore, there appears to be both an effect and latency in the diffusion of knowledge concerning the management of thyroid nodules.

TSH assay must be performed as first-line assessment of thyroid disease, as its sensitivity allows the detection of all cases of thyroid dysfunction. ${ }^{10} \mathrm{~T} 4$ assay should only be requested as second-line test, together with T3 when TSH is low, and together with anti-thyroid peroxidase (TPO) antibody assay when TSH is high. ${ }^{10}$ However, in 2010, T4 assay was performed in almost two-thirds of patients and T3 assay in more than one-third of patients prior to thyroidectomy with thyroid cancer or benign nodule. Similarly, thyroid scintigraphy is no longer indicated in euthyroid patients, ${ }^{10}$ but it was performed prior to thyroidectomy in almost one in five patients who had thyroid cancer or benign nodule.

On the other hand, thyroid ultrasonography is an essential part of the diagnostic work-up. ${ }^{8-10} 14$ It was frequently performed in our study, but only in $80-84 \%$ of cases rather than $100 \%$ of cases, as other diagnostic examinations may have been performed: neck vessel ultrasonography, neck CT scan or MRI, for example, which also suggests the possibility of incidentalomas, that is, incidentally discovered nodules. The Tirads score, proposed in $2009,{ }^{15}$ can be used to evaluate the ultrasound risk of malignancy by taking six features into account. Based on a series of 4550 operated nodules, the sensitivity of the Tirads score to detect malignant lesions varied from $87 \%$ to $95 \%$, with a negative predictive value of $99 \%$, and an excellent interobserver reproducibility. ${ }^{3} 15$

Therefore, fine-needle aspiration cytology is recommended for all nodules associated with a high-risk context according to the Tirads score, all suspicious nodules or nodules larger than $2 \mathrm{~cm}$, before deciding on the indication for surgery. ${ }^{10}$ However, fine-needle aspiration cytology was performed in less than one-half of patients operated with a diagnosis of thyroid cancer $(44 \%)$ or benign nodule $(34 \%)$.

The percentage of patients receiving postoperative levothyroxine replacement therapy appears to be consistent with the surgical procedure performed, as this rate was $99 \%$ among patients who had undergone total thyroidectomy. Levothyroxine therapy rates after partial thyroidectomy were $73 \%$ in the thyroid cancer group and $44 \%$ in the nodule group. The expected hypothyroidism rate after partial thyroidectomy has been estimated by others to be $11 \%,{ }^{16}$ but this figure must be adjusted to the volume of thyroid parenchyma left, which is not available in our study. T4 assay was performed at least once in more than two-thirds of patients. At least one T3 assay was performed in more than one-fourth of patients after surgery. Although a proportion of patients require adjustment of thyroid hormone replacement therapy, T3 assay appears to be inappropriate and generates an excess cost.

This excess cost must be added to sick leave allowances received by employed patients. Guidelines for practitioners were introduced in France in 2010 to recommend sick leave of 10-15 days after thyroidectomy. In our study, more than $85 \%$ of patients received sick leave allowances for more than 14 days and more than $60 \%$ received sick leave allowances for more than 21 days. However, the specific reasons for extension of sick leave (ie, difficulties adjusting replacement therapy, complications of surgery or other causes) are not recorded in the Sniiram database.

The postoperative or short-term mortality rates and the rate of compressive haematoma requiring reoperation, $0.1 \%$ and $0.4 \%$, respectively, are situated in the lower end of the ranges reported in the literature. The postoperative bleeding rate reported in the literature ranges from $0 \%$ to $6.5 \% .^{17}$ In 2014, Weiss et $a l^{19}$ reported a compressive haematoma rate of $1.25 \%$ in a series of 150012 patients. Compressive haematoma is associated with a 2.9-fold increased risk of mortality, as mortality rates were $1.34 \%$ in the presence of haematoma versus $0.32 \%$ for the overall group. Some authors consider that thyroid cancer is associated with an increased risk of haematoma, ${ }^{20}$ but such an association was not observed in our cohort. Compressive haematoma can be life-threatening and requires emergency decompression. It can also occur beyond the sixth postoperative hour. The Association Francophone de Chirurgie Endocrinienne (AFCE) has recently recommended that total thyroidectomy should not be performed as an ambulatory procedure. ${ }^{21}$

Hypoparathyroidism and recurrent laryngeal nerve injuries were the most common complications of thyroidectomy. In the present study, the estimated hypoparathyroidism rate between 4 months and 1 year after surgery was $6 \%$ in the thyroid cancer group and $1 \%$ in the benign nodule group, on the basis of more than three serum calcium assays and more than three deliveries of calcium supplements. However, it increased up to $3 \%$ in the benign nodule group when total thyroidectomy was performed. The recurrent laryngeal nerve injury rate is more difficult to estimate: $9 \%$ and $4 \%$ of patients in the two groups attended speech therapy sessions, and $23 \%$ and $17 \%$ attended at least two ENT visits or speech therapy sessions or underwent functional testing for recurrent laryngeal nerve injuries during the first 12 postoperative months. The complication rate depends on the time since the operation, the mode of detection and the definition of postoperative 
complications. For example, Duclos et $a l,{ }^{22}$ using a serum calcium cut-off of $2 \mathrm{mmol} / \mathrm{L}$, reported postoperative hypoparathyroidism and permanent hypoparathyroidism rates of $25.9 \%$ and $2.7 \%$, respectively. The unilateral or bilateral recurrent laryngeal nerve injury rate varies according to the time since the operation, $2.3 \%$ after 1 year versus $9.8 \%$ immediately postoperatively, and the mode of detection, $<2 \%$ without specific examination versus more than $6 \%$ when indirect laryngoscopy is performed. ${ }^{23}$ Our estimations are based on an indirect approach, which can overestimate or underestimate the true complication rate.

\section{CONCLUSION}

With more than 35400 general scheme beneficiaries (or about 45800 nationwide) who underwent surgery in 2010, and 35300 in 2013, thyroidectomy is one of the surgical procedures most commonly performed in France. The thyroidectomy rate with a diagnosis of benign nodule appears to be excessively high compared with the thyroidectomy rate with a diagnosis of thyroid cancer. Such assessment is likely to be shared by many European countries. ${ }^{7}$ Partial compliance with guidelines prior to thyroidectomy, especially the low rate of fine-needle aspiration cytology, indicates the need for large-scale diffusion of current guidelines and clinical practice evaluation by all professionals involved in the care pathway.

Several actions have been initiated by the French national health insurance in 2015 to reduce potential harms from overdiagnosis and overtreatment. Specific booklets have been developed for general practitioners and patients. ${ }^{24}$ Dedicated visits to general practitioners, specialists and surgeons are performed. Public or private hospitals with a low ratio of thyroidectomies with a diagnosis of cancer over thyroidectomies with a diagnosis of benign nodule are monitored.

Active surveillance of microcarcinomas with a rigorous patient selection is also being discussed among international experts. ${ }^{25}$ It involves a psychological cost to the patient and a financial cost to the society due to the annual follow-up (medical visit, thyroid and lymph node ultrasound scan) and should be discussed with each patient. Some French experts recommend practising fine-needle aspiration of a micronodule in case of suspicion of lymph node or extrathyroid involvement, or of a documented increasing size of the nodule, if the micronodule is based on the isthmus or at the highest third part of the thyroid, or if the patient is aged $<40$ years. An international review of the relevance of thyroidectomy and assessment of the long-term risk of microcancers is necessary in view of changing international clinical practices. $^{725}$

\footnotetext{
Author affiliations

${ }^{1}$ Hospital of Limoges, Limoges, France

${ }^{2}$ Caisse Nationale d'Assurance Maladie des Travailleurs Salariés, Paris, France

${ }^{3}$ Department of General, Visceral and Endocrine Surgery, Pitié-Salpêtrière

Hospital, Paris, France
}

${ }^{4}$ Unit of Gynecology and Endocrinology, Cochin Hospital, Paris, France ${ }^{5}$ Ministry of Health, Paris, France

${ }^{6}$ Department of Thyroid Diseases and Endocrine Tumors, Pitié Salpêtrière Hospital, University Paris VI, Institut E3M, Paris, France

${ }^{7}$ Department of Pathology, La Pitié Salpétrière Hospital, Paris, France

${ }^{8}$ Lapeyronie Hospital, Paris, France

Contributors MM, AC, CT, J-CT, EF-L, PT, BM and AF-C were involved in study concept and design. MM, AC, CT, J-CT, EF-L, GR, LL, CB, PT, BM and $A F-C$ were involved in interpretation of data. MM, $A C$ and $A F-C$ were involved in drafting of the manuscript. MM, AC, CT, J-CT, EF-L, GR, LL, CB, PT, BM and $A F-C$ were involved in critical revision of the manuscript for important intellectual content. AC was involved in statistical analysis. MM, BM and AF-C were involved in study supervision.

Funding This research received no specific grant from any funding agency in the public, commercial or not-for-profit sectors.

Competing interests None declared.

Ethics approval Commission nationale informatique et libertes (CNIL).

Provenance and peer review Not commissioned; externally peer reviewed.

Data sharing statement No additional data are available.

Open Access This is an Open Access article distributed in accordance with the Creative Commons Attribution Non Commercial (CC BY-NC 4.0) license, which permits others to distribute, remix, adapt, build upon this work noncommercially, and license their derivative works on different terms, provided the original work is properly cited and the use is non-commercial. See: http:// creativecommons.org/licenses/by-nc/4.0/

\section{REFERENCES}

1. Dean DS, Gharib H. Epidemiology of thyroid nodules. Best Pract Res Clin Endocrinol Metab 2008;22:901-11.

2. Mazzaferri EL. Management of a solitary thyroid nodule. N Engl J Med 1993;238:553-9.

3. Russ G, Leboulleux S, Leenhardt L, et al. Thyroid incidentalomas: epidemiology, risk stratification with ultrasound and workup. Eur Thyroid J 2014;3:154-63.

4. Ezzat S, Sarti DA, Cain DR, et al. Thyroid incidentalomas. Prevalence by palpation and ultrasonography. Arch Intern Med 1994;154:1838-40.

5. Colonna M, Bossard N, Guizard AV, et al. Descriptive epidemiology of thyroid cancer in France: incidence, mortality and survival. Ann Endocrinol 2010;71:95-101.

6. Colonna M, Guizard AV, Schvartz C, et al. A time trend analysis of papillary and follicular cancers as function of tumour size: a study of data from six cancers registries in France (1983-2000). Eur J Cancer 2007;43:891-900.

7. Brito JP, Morris JC, Montori VM. Thyroid cancer: zealous imaging has increased detection and treatment of low risk tumours. BMJ 2013;347:f4706

8. Gharib H, Papini E, Paschke R. Thyroid nodules: a review of current guidelines, practices, and prospects. Eur J Endocrinol 2008;159:493-505.

9. Paschke R, Hegedüs L, Alexander E, et al. Thyroid nodules guidelines: agreement, disagreement and need for future research. Nat Rev Endocrinol 2011;7:354-61.

10. Wémeau JL, Sadoul JL, d'Herbomez M, et al. Recommandations de la Société Française d'Endocrinologie pour la prise en charge des nodules thyroïdiens. Presse Med 2011;40:793-826.

11. Tuppin $\mathrm{P}$, de Roquefeuil $\mathrm{L}$, Weill $\mathrm{A}$, et al. French national health insurance information system and the permanent beneficiaries sample. Rev Epidemiol Sante Publique 2010;58:286-90.

12. Maura G, Blotière $\mathrm{PO}$, Bouillon $\mathrm{K}$, et al. Comparison of the short-term risk of bleeding and arterial thromboembolic events in nonvalvular atrial fibrillation patients newly treated with dabigatran or rivaroxaban versus vitamin $\mathrm{K}$ antagonists: a French nationwide propensity-matched cohort study. Circulation 2015;132:1252-60.

13. Weill A, Dalichampt M, Raguideau F, et al. Low dose oestrogen combined oral contraception and risk of pulmonary embolism, stroke, and myocardial infarction in five million French women: cohort study. BMJ 2016;353:i2002.

14. Horvath E, Majlis S, Rossi R, et al. An ultrasonogram reporting system for thyroid nodules stratifying cancer risk for clinical management. J Clin Endocrinol Metab 2009;94:1748-51. 
15. Russ G, Royer B, Bigorgne C, et al. Prospective evaluation of thyroid imaging reporting and data system on 4550 nodules with and without elastography. Eur J Endocrinol 2013;168:649-55

16. Su SY, Grodski S, Serpell JW. Hypothyroidism following hemithyroidectomy: a retrospective review. Ann Surg 2009:250:991-4.

17. Calò PG, Pisano G, Piga G, et al. Postoperative hematoma after thyroid surgery. Incidence and risks factors in our experience. Ann Ital Chir 2010;81:343-7.

18. Leyre P, Desurmont T, Lacoste L, et al. Does the risk of compressive hematoma after thyroidectomy authorize 1-day surgery ? Langenbecks Arch Surg 2008;393:733-7.

19. Weiss A, Lee KC, Brumund KT, et al. Risk factors for hematoma after thyroidectomy: results from the nationwide inpatient sample. Surgery 2014;156:399-404.

20. Promberger R, Ott J, Kober F, et al. Risk factors for postoperative bleeding after thyroid surgery. Br J Surg 2012;99:373-9.
21. Menegaud F. Ambulatory thyroidectomy: recommendations from the Association Francophone de Chirurgie Endocrinienne (AFCE). Investigating current practices. J Visc Surg 2013;150:165-71.

22. Duclos A, Peix JL, Colin C, et al. Influence of experience on performance of individual surgeons in thyroid surgery: prospective cross-sectional multicentre study. BMJ 2012;344:d8041. doi:10.1136/BMJD8041

23. Jeannon JP, Orabi AA, Bruch GA, et al. Diagnosis of recurrent laryngeal nerve palsy after thyroidectomy: a systematic review. Int J Clin Pract 2009;63:624-9.

24. http://www.ameli.fr/professionnels-de-sante/medecins/ exercer-au-quotidien/aide-a-la-pratique-memos/

les-memos-de-bonne-pratique/nodule-thyroidien-sans-hyperthyroidie. php, consulted 12/28/2015.

25. Leboulleux S, Tuttle RM, Pacini F, et al. Papillary thyroid microcarcinoma: time to shift from surgery to active surveillance? Lancet Diabetes Endocrinol 2016;4:933-42. doi: 10.1016/S22138587(16)30180-2. Epub 2016 Aug 20. 